\title{
ON IDEALS OF THE COEFFICIENT RINGS IN GROUP RINGS
}

\author{
SUSHMA SAINI
}

\begin{abstract}
A bstract. Let $R$ and $S$ be rings, $G$ any group. If the group rings $R G$ and $S G$ are isomorphic as rings, we formulate a correspondence between the ideals of $R$ and those of $S$ and show that this correspondence is one-to-one in case $R$ and $S$ are isomorphic. It is shown that this correspondence also works for Jordan ideals, provided that $G$ is abelian.
\end{abstract}

\section{Introduction}

In the study of group rings, one comes across two types of isomorphism problems. The first isomorphism problem asks as to what extent the group ring $R G$ determines the group $G$. The answer in general is no. The second isomorphism problem asks whether or not $R$ is an invariant of $R G$. More precisely if for any two rings $R$ and $S$ and a fixed group $G, R G$ isomorphic to $S G$ as a ring, does it follow that $R$ is isomorphic to $S$ ? The answer is again no in general. While studying the first isomorphism problem, a normal subgroup correspondence has been established (e.g. see [1], [4], [7], [8]). We take up the case when $R G \cong S G$ and formulate a correspondence between the ideals (i.e. two sided ideals) of $R$ and $S$. We also show that this correspondence is one-to-one in case the second isomorphism problem is resolved. Finally we show that this correspondence also works for Jordan ideals (Lie ideals), provided that $G$ is an abelian group.

\section{Ideal-Correspondence}

Proposition 2.1. Let $R$ and $S$ be rings, $G$ any group and $\theta: R G \cong S G$ a ring isomorphism. For every ideal $I$ of $R$, set

$$
\phi(I)=\left\{s \in S \mid s 1_{G} \in \theta(I G)\right\}
$$

Received November 11, 1991.

1991 Mathematics Subject Classification. 16S34.

Key words and phrases. Group ring, ring isomorphism, lattice of ideals, torsion ideal, Jordan ideal, nil-ideal. 
Then

(i) $\phi(I)$ is an ideal of $S$ and for $I=R, \phi(I)=S$

Thus we have a correspondence

$$
\phi: \mathcal{L}_{g}(R) \longrightarrow \mathcal{L}_{g}(S)
$$

where $\mathcal{L}_{g}(R)$ and $\mathcal{L}_{g}(S)$ denote lattices of ideals of $R$ and $S$ respectively.

Similarly we have a correspondence

$$
\psi: \mathcal{L}_{g}(S) \longrightarrow \mathcal{L}_{g}(R)
$$

(ii) If $\theta$ induces an isomorphism between $R$ and $S$, we have

$$
\phi \psi=1, \quad \psi \phi=1
$$

i.e. $\phi$ is a one-to-one correspondence.

Proof. (i) For any ideal $I$ of $R, I G$ is an ideal of $R G$ and as $\theta$ is a ring homomorphism of $R G$ onto $S G, \theta(I G)$ is an ideal of $S G$.

$$
\begin{gathered}
\because O_{S} 1_{G} \in \theta(I G) \Longrightarrow O=O_{S} \in \phi(I) \\
\therefore \phi(I) \text { is a non-empty subset of } S .
\end{gathered}
$$

Now take any $s, t \in \phi(I)$. Then $s 1_{G} \in \theta(I G), t 1_{G} \in \theta(I G)$ Since $\theta(I G)$ is an ideal of $S G, s 1_{G}-t 1_{G} \in \theta(I G)$, so $(s-t) 1_{G} \in \theta(I G)$ and hence $s-t \in \phi(I)$.

This proves that $\phi(I)$ is an additive subgroup of $S$. Now take any $t \in \phi(I)$ and $s \in S$. We again use the fact that $\theta(I G)$ is an ideal of $S G$ to get $\left(t 1_{G}\right)\left(s 1_{G}\right) \in \theta(I G)$ and $\left(s 1_{G}\right)\left(t 1_{G}\right) \in \theta(I G)$.

$$
\begin{gathered}
\because(t s) 1_{G} \in \theta(I G) \text { and }(s t) 1_{G} \in \theta(I G) \\
\Longrightarrow t s \in \phi(I) \text { and } s t \in \phi(I) . \\
\therefore \phi(I) \text { is an ideal of } S .
\end{gathered}
$$

Clearly for $I=R, \phi(I)=S$.

(ii) Since $\phi(I)$ is an ideal of $S$,

$\therefore \phi(I) G$ is an ideal of $S G$.

Take any element $\sum s_{g} g \in \phi(I) . G, s_{g} \in \phi(I) \forall g \in G$. 
Then

$$
\begin{aligned}
& \theta^{-1}\left(\sum s_{g} g\right)=\theta^{-1}\left(\sum\left(s_{g} 1_{G}\right)\left(1_{s} g\right)\right) \\
&=\sum \theta^{-1}\left[\left(s_{g} 1_{G}\right)\left(1_{s} g\right)\right] \\
&=\sum \theta^{-1}\left(s_{g} 1_{G}\right) \theta^{-1}\left(1_{s} g\right) \\
& \because s_{g} \in \phi(I), \quad \therefore s_{g} 1_{G} \in \theta(I G) .
\end{aligned}
$$

This implies that $\theta^{-1}\left(s_{g} 1_{G}\right) \in I G$.

Also $\theta^{-1}\left(1_{s} g\right) \in R G$ and since $I G$ is an ideal of $R G$

$$
\begin{aligned}
& \therefore \theta^{-1}\left(s_{g} 1_{G}\right) \theta^{-1}\left(1_{s} g\right) \in I G \\
\Longrightarrow & \sum \theta^{-1}\left(s_{g} 1_{G}\right) \theta^{-1}\left(1_{-S} g\right) \in I G \\
\Longrightarrow & \theta^{-1}\left(\sum s_{g} g\right) \in I G .
\end{aligned}
$$

Thus we see that

$$
\theta^{-1}(\phi(I) G) \subseteq I G
$$

Consider $\psi: \mathcal{L}_{g}(S) \longrightarrow \mathcal{L}_{g}(R)$. Then

$\psi(J)=\left\{r R \mid r 1_{G} \in \theta^{-1}(J G)\right\}$ for every ideal $J$ of $S$.

Claim $\phi \psi=1, \psi \phi=1$

For any ideal $I$ of $R$, consider $\psi \phi(I)$. Take any $r \in \psi \phi(I)$. Then $r \in R$ and $r 1_{G} \in$ $\theta^{-1}(\phi(I) G)$ i.e. $r \in R$ and $r 1_{G} \in I G$ (using (1)), hence $r \in I$.

$\therefore \psi \phi(I) \subseteq I$.

Conversely take any $r \in I$. Then $r \in \psi \phi(I)$ iff $r 1_{G} \in \theta^{-1}(\phi(I) G)$ i.e. iff $\theta\left(r 1_{G}\right) \in \phi(I) G$. Now as $\theta$ induces an isomorphism between $R$ and $S$,

$\therefore \theta\left(r 1_{G}\right)=s 1_{G}$ for some $s \in S$, and so $s \in \phi(I) \quad\left(\because s 1_{G} \in \theta(I G)\right)$.

$\therefore \theta\left(r 1_{G}\right) \in \phi(I) G$

$\Longrightarrow r .1_{G} \in \theta^{-1}(\phi(I) G)$

$\Longrightarrow r \in \psi \phi(I)$.

$\therefore I \subseteq \psi \phi(I)$

$\therefore \psi \phi=1$

Similarly $\phi \psi=1$.

Hence $\phi$ is a one-to-one correspondence provided that $\theta$ induces an isomorphism between $R$ and $S$.

Remark 2.2. There are many instances when $\theta$ does induce an isomorphism between $R$ and $S$. We mention a few:

(i) If $R$ and $S$ are P.I.D's which do not contain fields and $\langle x\rangle$ is an infinite cyclic group then $R<x\rangle \cong S<x>$, implies that $R \cong S([5])$.

(ii) If $R$ and $S$ are integral domains and $G$ is a torsion abelian group then $R G \cong S G$ implies that $R \cong S([6])$. 
Example 2.3. Now we give an example to show that in case $\theta: R G \cong S G$ does not induce an isomorphism from $R$ to $S$, the correspondence obtained by us need not be a one-to-one correspondence.

Let $G_{0}, G_{1}, G_{2}, \ldots$ be infinite cyclic groups and $R=Z, S=Z G_{0}$ and $G=\prod_{i \geq 1} G_{i}$, the direct product of $G_{1}, G_{2}, \ldots$ Then we have an isomorphism $\theta: R G \cong S G$ which does not induce an isomorphism between $R$ and $S$.

Take $J=\Delta_{Z}\left(G_{0}\right)$, the augmentation ideal of $Z G_{0} . J$ is an ideal of $S$ but there does not exist any ideal $I$ of $R$ such that $J=\phi(I)$.

Thus the correspondence $\phi$ is not a bijective one.

Now we study a few properties of the correspondence $\phi$ (irrespective of the fact that it is bijective or not).

Proposition 2.4. (i) If $I$ is a nilpotent ideal of $R$, then $\phi(I)$ is a nilpotent ideal of $S$.

(ii) Suppose $R$ is a commutative ring without proper zero divisors and $I$ is a torsion ideal of $R$, then $\phi(I)$ is a torsion ideal of $S$.

(iii) Suppose $G$ is an abelian group, $R$ a commutative ring and $I$ is a nil-ideal of $R$, then $\phi(I)$ is a nil-ideal of $S$.

Proof. (i) Since $I$ is a nilpotent ideal of $R, I^{n}=0$ for some integer $n>1$.

Now $(I G)^{n} \subseteq I^{n} G=0=0_{R} 1_{G}$ It follows that $(\theta(I G))^{n}=0$. Take any $s_{1}, s_{2}, \ldots, s_{n} \in \phi(I)$. Then $s_{1}, s_{2}, \ldots, s_{n} \in S$ and $s_{1} 1_{G}, s_{2} 1_{G}, \ldots, s_{n} 1_{G} \in \theta(I G)$. Since $(\theta(I G))^{n}=0, s_{1} 1_{G} \cdot s_{2} 1_{G} \cdots s_{n} 1_{G}=0$. This implies $\left(s_{1} s_{2} \cdots s_{n}\right) 1_{G}=01_{G}$, hence $s_{1} s_{2} \cdots s_{n}=0$

Thus $(\phi(I))^{n}=0$ and so $\phi(I)$ is a nilpotent ideal of $S$.

(ii) Take any $r_{1} g_{1}+r_{2} g_{2}+\ldots+r_{n} g_{n} \in I G, r_{1}, r_{2}, \ldots r_{n} \in I ; g_{1}, g_{2}, \ldots, g_{n} \in G$. Since $I$ is a torsion ideal of $R$, there exist $t_{1}, t_{2}, \ldots, t_{n} \in R$ such that $t_{i} r_{i}=0$ ( $i=$ $1,2, \ldots, n, t_{i} \neq 0$ ). Then $t=t_{1} t_{2} \cdots t_{n} \neq 0$ (as $R$ is without zero divisors) and $\left(t 1_{G}\right)\left(r_{1} g_{1}+r_{2} g_{2}+\ldots+r_{n} g_{n}\right)=0$, so $I G$ is a torsion ideal of $R G$.

Now take any $\theta(\xi) \in \theta(I G), \xi \in I G$. Since $I G$ is a torsion ideal of $R G$, there exists $\eta \in R G, \eta \neq 0$ such that $\eta \xi=0$. Then $\theta(\eta \xi)=0$ i.e. $\theta(\eta) \theta(\xi)=0$. As $\theta$ is $1-1$ and $\eta \neq 0, \theta(\eta) \neq 0$ and so we see that $\theta(I G)$ is a torsion ideal of $S G$.

Finally take any $s \in \phi(I)$. Then $s \in S$ and $s 1_{G} \in \theta(I G)$. Since $\theta(I G)$ is a torsion ideal of $S G$, there exists $s_{1} g_{1}+s_{2} g_{2}+\ldots+s_{n} g_{n} \neq 0 \in S G\left(g_{1}, g_{2}, \ldots, g_{n}\right.$ distinct elements of $G$ ) such that $\left(s_{1} g_{1}+s_{2} g_{2}+\ldots+s_{n} g_{n}\right)\left(s 1_{G}\right)=0$. This implies that $s_{1} g_{1}+s_{2} g_{2}+\ldots+s_{n} g_{n}=0$.

$$
\begin{gathered}
\therefore s_{1} s=0, s_{2} s=0, \ldots, s_{n} s=0 \\
\because s_{1} g_{1}+s_{2} g_{2}+\ldots+s_{n} g_{n} \neq 0, \therefore s_{j} \neq 0 \text { for some } j(1 \leq j \leq n) .
\end{gathered}
$$

$\therefore$ We see that for every $s \in \phi(I)$, there exists $s_{j} \in S, s_{j} \neq 0$ such that $s_{j} s=0$. Hence $s$ is a torsion element of $S$. 
$\therefore \phi(I)$ is a torsion ideal of $S$.

(iii) Since $I$ is a nil-ideal of $R$ and $R G$ is commutative, $I G$ is a nil-ideal of $R G$. Take any $\theta(\xi) \in \theta(I G) ; \xi \in I G$. Since $I G$ is a nil-ideal of $R G$, there exists an integer $n>1$ such that $\xi^{n}=0$. Now $(\theta(\xi))^{n}=\theta\left(\xi^{n}\right)=\theta(0)=0 \therefore \theta(I G)$ is a nil-ideal of $S G$. Take any $s \in \phi(I)$. Then $s \in S$ such that $s 1_{G} \in \theta(I G)$. Since $\theta(I G)$ is a nilideal of $S G$, there exists an integer $n>1$ such that $\left(s 1_{G}\right)^{n}=0_{S} 1_{G}$ i.e. $s^{n} 1_{G}=0_{S} 1_{G}$ and so we must have $s^{n}=0$. Thus $s$ is nilpotent and $\phi(I)$ is shown to be a nil-ideal of $S$.

\section{Jordan ideal Correspondence}

In what follows, we assume that $G$ is an abelian group. We show that given any ring isomorphism $\theta: R G \cong S G$ of group rings, to every Jordan ideal (Lie ideal) of $R$, there corresponds a Jordan ideal (Lie ideal) of $S$.

Definition 3.1. ([2],[3]) An additive subgroup $U$ of a ring $R$ is said to be a Jordan ideal of $R$ if whenever $u \in U$ and $r \in R$, then $u \circ r=u r+r u$ is in $U$.

Definition 3.2. ([2],[3]) An additive subgroup $U$ of a $\operatorname{ring} R$ is said to be a Lie ideal of $R$ if whenever $u \in U$ and $r \in R$, then $[u, r]=u r-r u$ is in $U$.

Remark 3.3. ([2]) Every two sided ideal is a Jordan (Lie) ideal but converse is not true in general.

Now we proceed to show that if $\theta: R G \cong S G$ and $I$ is a Jordan ideal of $R$, then $\phi(I)$ is a Jordan ideal of $S$, where $\phi(I)=\left\{s \in S \mid s 1_{G} \in \theta(I G)\right\}$.

If $I$ is a Jordan ideal of $R$, it is not difficult to show that $I G$ is a Jordan ideal of $R G$.

Take any arbitrary element $\xi \in \theta(I G)$ and $\eta \in S G$. Then $\xi=\theta(\alpha)$ for some $\alpha \in I G$. Also as $\theta$ is on-to, there exists $\beta \in R G$ such that $\theta(\beta)=\eta$

Then

$$
\begin{aligned}
& \xi \circ \eta=\xi \eta+\eta \xi \\
= & \theta(\alpha) \theta(\beta)+\theta(\beta) \theta(\alpha) \\
= & \theta(\alpha \beta)+\theta(\beta \alpha) \\
= & \theta(\alpha \beta+\beta \alpha) \in \theta(I G) \quad(\alpha \beta+\beta \alpha \in I G \text { as } I G \text { is a Jordan ideal of } R G)
\end{aligned}
$$

$\therefore \theta(I G)$ is a Jordan ideal of $S G$.

Now to see that $\phi(I)$ is a Jordan ideal of $S$, it suffices to show that for $t \in \phi(I)$ and $s \in S, t s+s t \in \phi(I)$.

$$
t \in \phi(I) \Longrightarrow t \in S \text { and } t 1_{G} \in \theta(I G) .
$$


Also $s 1_{G} \in S G$ and since $\theta(I G)$ is a Jordan ideal of $S G$,

$$
\begin{aligned}
& \left(t 1_{G}\right)\left(s 1_{G}\right)+\left(s 1_{G}\right)\left(t 1_{G}\right) \in \theta(I G) \\
\Longrightarrow & \left(t s 1_{G}\right)+\left(s t 1_{G}\right) \in \theta(I G) \\
\Longrightarrow & (t s+s t) 1_{G} \in \theta(I G) \\
\Longrightarrow & t s+s t \in \phi(I)
\end{aligned}
$$

$\therefore \phi(I)$ is a Jordan ideal of $S$.

Essentially the same argument as above gives that if $I$ is a Lie ideal of $R$, then $\phi(I)$ is Lie ideal of $S$.

\section{Acknowledgement}

I would like to express my deep sense of gratitude towards my research supervisor Dr. (Miss) Sneh Sharma for her constant guidance throughout the preparation of this paper.

\section{References}

[1] Toru Furukawa, "Isomorphism of group rings of infinite nilpotent groups," Osaka J. Math., 24 (1987), 95-105.

[2] I.N. Herstein, "Lie and Jordan structures in simple associative rings," Bull. Amer. Math. Soc., 67 (1961), 517-531.

[3] I.N. Herstein, Topics in ring theory, the University of Chicago Press, 1969.

[4] G. Karpilovsky, "On certain group ring problems," Bull. Austral. Math. Soc., Vol. 21(1980), 329350. [5] M.M Parmenter, "Units and isomorphism in group rings," Quaestioner Mathematicae, 8(1985),
9-14.

[6] M.M. Parmenter and E. Spiegel, "Isomorphic group rings over domains revisited," Journal of Algebra, (129), 518-523(1990).

[7] R. Sandling, "The isomorphism problem for group rings: a survey," Lecture Notes in Math., Springer, Berlin, 1985.

[8] S.K. Sehgal, Topics in group rings, Marcel Dekker, New York, 1978.

Department of Mathematics, Guru Nanak Dev University, Amritsar-143005, India. 